\title{
General stability and exponential growth of nonlinear variable coefficient wave equation with logarithmic source and memory term
}

\author{
Long Yan $^{1}$ and Lili Sun ${ }^{1}$ \\ ${ }^{1}$ Northeast Electric Power University
}

September 24, 2021

\begin{abstract}
This paper is concerned with the asymptotic stability and instability of solutions to a variable coefficient logarithmic wave equation with nonlinear damping and memory term. This model describes wave travelling through nonhomogeneous viscoelastic materials. By choosing appropriate multiplier and using weighted energy method, we prove the exponential decay of the energy. Besides, we also obtain the instability at the infinity of the solutions in the presence of the nonlinear damping.
\end{abstract}

\section{Hosted file}

viscoelastic logarithmic wave equation--R2.pdf available at https://authorea.com/users/ 434898/articles/538288-general-stability-and-exponential-growth-of-nonlinear-variablecoefficient-wave-equation-with-logarithmic-source-and-memory-term 